\title{
Comparison of fecal pooling strategies for detection of Mycobacterium avium ssp. paratuberculosis in cattle
}

\author{
S. L. B. McKenna, ${ }^{* 1}$ C. Ritter, $\dagger^{1}$ I. Dohoo, ${ }^{*}$ G. P. Keefe, ${ }^{*}$ and H. W. Barkema ${ }^{2}$ \\ *Department of Health Management, University of Prince Edward Island, Charlottetown, PE, Canada C1A 4P3 \\ †Department of Production Animal Health, University of Calgary, Calgary, AB, Canada T2N 4N1
}

\begin{abstract}
In herds with typical moderate to low within-herd prevalence, testing for Mycobacterium avium ssp. paratuberculosis (MAP), the infectious agent of Johne's disease, will be more cost-effective if individual fecal samples are cultured in composite pools. However, sensitivity to classify a pool containing 1 or more positive individual samples as positive may depend on pool size and number of individual positive samples within a pool. Fecal samples collected from 994 dairy cows sampled at slaughter were cultured to detect MAP. Culturing was done both individually and as composite pooled samples using the TREK ESP Culture System II broth medium (Thermo Fisher Scientific, Trek Diagnostic Systems Inc., Cleveland, $\mathrm{OH}$ ). Composite samples consisted of pools containing feces from $3,5,8,10$, or 15 cows. The number of individual fecal culture-positive cows within each pool ranged from 0 to 4 . Culture of individual fecal samples detected MAP in $36(3.6 \%)$ of the 994 cows. Individual samples that were detected within the first $50 \mathrm{~d}$ by TREK ESP Culture System II were more likely to lead to a positive pool result. In total, 840 pooled fecal samples were examined for presence of MAP, and of those, 272 pools actually contained feces from fecal culture-positive cows. The crude sensitivity (proportion of pools that contained at least 1 fecal-positive cow that tested positive) for pools of $3,5,8,10$, and 15 was 47, 67, 44, 59, and 39\%, respectively. Across pools, an increase of the number of fecal culture-positive samples from 1 to 2 enhanced overall crude sensitivity from 44 to $71 \%$. However, sensitivity did not further increase for pools with 3 or 4 fecal culture-positive samples (63 and $60 \%$, respectively). Additionally, a simulation analysis assessing probability of pooled fecal samples being positive in herds of 50 and 100 cows was conducted. The simulation assumed that 1,2 , or 5 cows per herd were
\end{abstract}

Received January 16, 2018.

Accepted April 14, 2018.

${ }^{1}$ These authors contributed equally to this work.

${ }^{2}$ Corresponding author: barkema@ucalgary.ca
MAP fecal culture-positive and that pools of 5 and 10 were used. This low-prevalence herd simulation indicated that weighted mean herd probabilities of detecting a positive herd ranged between 52 and $99.3 \%$, with the lowest probability for pools of 10 with 1 positive cow in the herd and the highest probability for pools of 5 with 5 positive cows in the herd. However, overall, pools of 5 and 10 had similar diagnostic capabilities, enabling cost savings by utilizing pools of 10 .

Key words: Mycobacterium avium ssp. paratuberculosis, pooling, sensitivity, fecal and tissue culture, Johne's disease

\section{INTRODUCTION}

Johne's disease (JD) in cattle is an infectious, chronic granulomatous enteritis caused by Mycobacterium avium ssp. paratuberculosis (MAP). The disease is widespread in cattle populations around the world and typically infects animals in the early stages of life. After a latent period without overt clinical signs, JD can cause decreased milk production, loss of body condition, and intermittent diarrhea (McKenna et al., 2006). Economic losses that the individual farmer and the dairy industry incur are due to decreased production and premature culling of infected cattle (Garcia and Shalloo, 2015). Furthermore, a causal association between Crohn's disease and MAP cannot be excluded (Barkema et al., 2010).

In addition to being an important individual-animal disease, JD is generally a herd problem. Identifying infected herds and implementing strategies to decrease prevalence and incidence in these herds, as well as avoiding transmission of MAP to uninfected herds, is the basic goal of most JD prevention and control programs (Barkema et al., 2017). Fecal culture is a frequent diagnostic tool to determine MAP infection status, with its primary advantage being high inherent specificity (close to 100\%), whereas a 38\% sensitivity was reported (Whitlock et al., 2000). However, the cost of fecal culture is a disadvantage (van Schaik et al., 2007). Therefore, pooling fecal samples in aggregates of 
various sizes has been suggested (Kalis et al., 2000; van Schaik et al., 2003, 2007; Weber et al., 2004).

An early investigation of culturing pooled fecal samples reported decreased sensitivity for detecting infected cattle in pools of 10 (Vialard et al., 1993). However, other studies have indicated that the sensitivity of a pooled fecal sample can be adequate. Sensitivity for pools of 10 cows was $69 \%$ (25 of 36 pools) from cows pre-selected based upon positive ELISA results (Tavornpanich et al., 2004). Using a modified Jorgensen culture method, sensitivity for pools of 5 cows that were strategically pooled by age was $81 \%$ (26 of 32 pools; Kalis et al., 2000). Two studies using Herrold's egg yolk medium culturing determined sensitivities ranging from 24 to $94 \%$, depending on pool size and bacterial concentrations (Wells et al., 2003; van Schaik et al., 2007). A Monte Carlo simulation for fecal culture diagnostics determined that pool sizes of 10 or 20 are recommended when suspected MAP prevalence ranges from 0.01 to 0.10 and test sensitivity was not expected to decrease with increased pool size (Messam et al., 2010).

Apparently, only 1 study investigated the use of the TREK ESP Culture System II (Thermo Fisher Scientific, Diagnostic Systems Inc., Cleveland, $\mathrm{OH}$ ) as a method to culture pooled samples (Tavornpanich et al., 2004). In that study, however, it could not be determined whether recovery of positive pools was different between the broth system and solid medium, because the study was limited to 87 samples per method [of which use of the TREK ESP Culture System II detected MAP in $50 \%$ (10 of 20) of positive pooled samples].

The objective of this study was, therefore, to determine sensitivity of fecal pooling using the TREK ESP Culture System II. Furthermore, we assessed whether sensitivity depended on pool size, number of individual culture-positive fecal samples within a pool, and time until detection of a positive individual fecal sample included in the pool (proxy for concentration of bacteria present). Finally, a simulation model was constructed to compare the use of pools of 5 and 10 in 2 herd sizes with varying degrees of low prevalence. The simulation aimed to determine the probability of a herd being correctly classified as being MAP infected, given various pool sizes and prevalence.

\section{MATERIALS AND METHODS}

\section{Sample Population}

Sample population and sample collection methods have been described (McKenna et al., 2004). In short, using a systematic random sample, 994 mature dairy cows from Atlantic Canada and Maine were selected at slaughter for collection of tissue samples and feces. Two lymph nodes in the mesenteric chain in the region of the ileum were collected, along with a 5 - to $10-\mathrm{cm}$ segment of the terminal ileum $\sim 25 \mathrm{~cm}$ proximal to the ileo-cecal junction. Fecal samples were collected from the posterior colon via an incision into the viscera. Samples were placed in individual containers labeled with the numeric identifier for each cow. Tissue cultures were performed initially and fecal samples were kept frozen at $-80^{\circ} \mathrm{C}$ until tissue culture results were available so that fecal pools could be assembled, such that all fecal pools (except 65 of 840) contained at least 1 tissue culture-positive cow. This process increased the likelihood that there would be adequate numbers of pools with fecal MAP culture-positive cows to have sufficient power to detect differences in herd level sensitivity. Strategic pooling by age cohorts, as recommended by Kalis et al. (2000), was not possible, due to a lack of cow age data.

\section{Tissue Preparation}

Tissue preparation for culture has been described (Wells et al., 2002a; McKenna et al., 2004). Briefly, samples were placed in a Tekmar bag along with $25 \mathrm{~mL}$ of $0.75 \%$ hexadecylpyridinium chloride (HPC) solution as a decontaminate. The sample was placed in a stomacher for a minimum of $1 \mathrm{~min}$, and then each sample was left undisturbed for 30 min to allow for separation of tissue. Then, a 10-mL sample of pulverized tissue mixture was transferred into another sterile tube containing a further $10 \mathrm{~mL}$ of HPC solution, used for the second decontamination stage. After a minimum of 3 $\mathrm{h}$ in the second decontamination stage, samples were centrifuged at $900 \times g$ for $30 \mathrm{~min}$ at room temperature. The supernatant was discarded and the pellet was resuspended with a combined half strength brain-heartinfusion (BHI) broth that contained $0.1 \%$ nalidixic acid (NAL), $0.1 \%$ vancomycin (VAN), and $0.05 \%$ amphotericin B (AMB; Allied Monitor Inc., Fayette, $\mathrm{MO}$ ). This mixture was vortexed and placed into sterile cryogenic tubes to be incubated for 12 to $14 \mathrm{~h}$ at $37^{\circ} \mathrm{C}$ to allow for growth of MAP and for antimicrobials to more effectively decrease levels of background contaminants. After the incubation phase, tubes were slowly cooled and then re-frozen at $-80^{\circ} \mathrm{C}$.

\section{Fecal Preparation}

For each cow, a 2-g sample of feces was weighed and placed in a $50-\mathrm{mL}$ centrifuge tube along with $35 \mathrm{~mL}$ of distilled deionized water. This mixture was vortexed and then placed on a shaker-table for $30 \mathrm{~min}$ to en- 
sure thorough mixing. Tubes were allowed to stand for $30 \mathrm{~min}$ to allow particles to settle, and then $5 \mathrm{~mL}$ of sample was drawn from the top third of the tube and transferred to a second $50-\mathrm{mL}$ centrifuge tube where it was mixed with $25 \mathrm{~mL}$ of $0.9 \%$ HPC solution mixed with half-strength $\mathrm{BHI}$ broth (final concentration of $0.75 \% \mathrm{HPC}$ ) as decontaminate. Sample tubes were then incubated at $37^{\circ} \mathrm{C}$ for $24 \mathrm{~h}$. Then each tube was placed in a new centrifuge tube and centrifuged at 900 $\times g$ for $30 \mathrm{~min}$ at room temperature. The supernatant was then discarded and the pellet re-suspended with a combined half strength BHI broth that contained $0.1 \%$ NAL, $0.1 \%$ VAN, and $0.05 \%$ AMB. This mixture was vortexed and placed into sterile cryogenic tubes, which were subsequently incubated for 12 to $14 \mathrm{~h}$ at $37^{\circ} \mathrm{C}$ similar to the tissue samples. Finally, samples were slowly cooled and then re-frozen at $-80^{\circ} \mathrm{C}$.

\section{Pooled Fecal Sample Preparation}

Based on results from the tissue culture that was initially performed, pools of feces were formulated in batches of $3,5,8,10$, and 15 samples per pool, at the same time as individual fecal testing. This was done by simple convenience, as fecal samples were being tested individually. As individual samples were being cultured, an assumption made was that previously known tissue culture MAP-positive cow samples would more likely be fecal culture-positive. This allowed for individual and pooled samples to be processed simultaneously, avoiding additional freezing and thawing of samples and subsequent potential loss of viable organisms. Some of the assumed positive cows were subsequently found negative, resulting in the matrix of actual distribution of positive cows among pools ranging from 0 to 5 (Table 1).

In constructing the pools, $2 \mathrm{~g}$ of feces from each cow designated in the pool was mixed using disposable plas- tic sticks and disposable weigh-boats. After thorough mixing to a homogeneous matrix, $2 \mathrm{~g}$ was weighed out for the pooled fecal culture. The same culture procedure was then used as described for the individual fecal samples.

\section{Culture Technique}

All samples were cultured using the TREK ESP Culture System II broth solution medium bottle (Thermo Fisher Scientific) according to manufacturer's user manual. Samples were thawed, and $1 \mathrm{~mL}$ from each of the tubes was inoculated into a TREK broth solution medium bottle, supplemented with egg yolk, antibiotics $(0.1 \% \mathrm{NAL}, 0.1 \% \mathrm{VAN}$, and $0.05 \% \mathrm{AMB})$, and a growth supplement containing Mycobactin J (Allied Monitor Inc.).

All sample bottles were then incubated for $56 \mathrm{~d}$, unless previously identified as positive by the TREK ESP Culture System II diagnostic incubator. As samples were identified as positive for bacterial growth by measurement of pressure changes within the bottle in the incubator, each bottle in turn was then examined for acid fast bacteria. After $56 \mathrm{~d}$, all remaining samples were also examined for acid fast bacteria, to identify samples that contained low numbers of bacteria unable to trigger the system. For this process, each bottle was vigorously shaken for a minimum of $60 \mathrm{~s}$ using a Mistral multi-mixer (Lab Line Instruments Inc., Melrose Park, IL).

A sample $(\sim 20 \mu \mathrm{L})$ from each bottle was placed on a microscope slide, with a total of 3 samples per slide. Slides were air-dried and then heat fixed and a cold Ziehl-Neelsen staining process was performed on each slide (Murray, 1999). Each slide was then examined at oil-immersion $(1,000 \times)$ for the presence of acid-fast bacteria. All samples that were acid fast-positive or suspicious were tested using a PCR technique.

Table 1. Number of pools by pool size and number of tissue and fecal culture-positive samples for Mycobacterium avium ssp. paratuberculosis within pools

No. of cows with culture-positive tissue samples in the pool

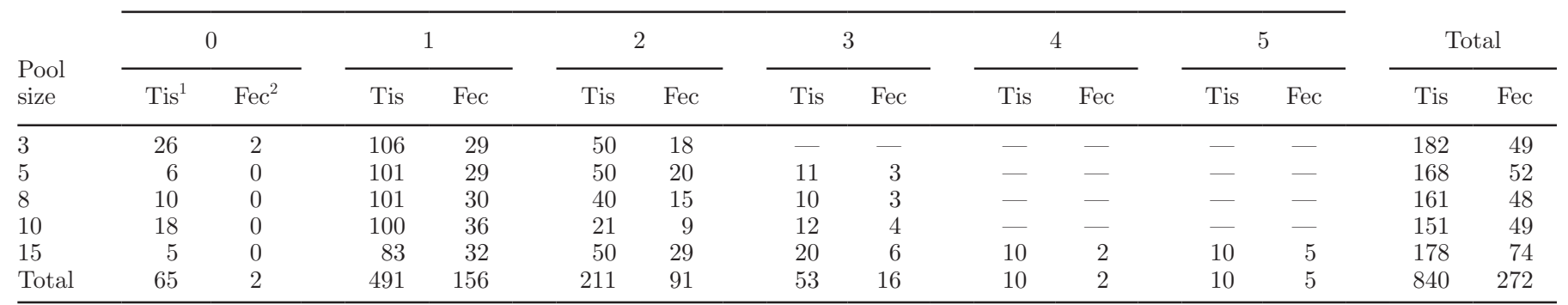

${ }^{1}$ Number of pools with 0 to 5 culture-positive tissue samples.

${ }^{2}$ Number of pools containing individual culture-positive fecal samples. 


\section{PCR Technique}

The procedure for PCR analysis has been described in detail (Kim et al., 2004; McKenna et al., 2004). Briefly, for DNA extraction, lysis by the boiling method was used by heating a mixture of sample with $100 \mu \mathrm{L}$ of lysis buffer containing HPLC-grade water with $1 \%$ Triton-X-100 (Fisher Scientific, Pittsburgh, PA), $1 \mathrm{~m} M$ EDTA, and $10 \mathrm{~m} M$ Tris-HCl (pH 8.0).

Primers used for the IS900 and F57 genetic elements have been described (Vary et al., 1990; Poupart et al., 1993). The forward primer designated IS900/150C and the reverse primer designated IS900/921 were used for IS900; the forward primer designated F57a and the reverse primer designated F57b were used for F57. Amplification reactions were performed on a total volume of $50 \mu \mathrm{L}$ containing $10 \mathrm{~m} M$ Tris- $\mathrm{HCl}(\mathrm{pH} 8.3) ; 1.5 \mathrm{~m} M$ $\mathrm{MgCl}_{2} ; 50 \mathrm{mM} \mathrm{KCl} ; 0.001 \%$ gelatin; $200 \mu M$ of each deoxynucleoside triphosphate (dATP, dCTP, dGTP, dTTP); $1 \mu M$ of each primer; $1.25 \mathrm{U}$ of Amplitaq (Applied Biosystems, Foster City, CA), and $5 \mu \mathrm{L}$ of boiled cell lysate for the monoplex PCR. The PCR assay was carried out in a Perkin-Elmer 2400 thermocycler (Perkin Elmer Corp., Norwalk, CT). A sample from a TREK ESP broth bottle containing MAP ATCC strain \#19698 was used as the DNA-positive control. The negative control consisted of a reaction mixture containing all reagents, but no DNA template. The PCR products were visualized by electrophoresis on a $1 \%$ agarose gel following standard procedures. Bacterial isolates positive to either F57 or IS900 genetic elements were classified as MAP-positive.

\section{Statistical Analyses}

The reference standard definition for a positive pool was as a pool that contained feces from at least 1 cow that was individually fecal culture-positive. A positive pool test result was a pooled sample from which MAP was isolated. Herd sensitivity was defined by identification of at least 1 positive pooled culture in a herd with 1 or more fecal culture-positive cows.

Time to positive detection in the individual samples was used to classify pooled samples containing 1 fecalpositive individual sample. This was done to correct for pools having a potential high shedder (expected to be early-detected) increasing the probability of being positive compared with the probability of a pool containing a low shedder.

Statistical analyses were performed using Stata IC 15.0 (Stata Corporation, College Station, TX) and Excel (2000, Microsoft Corporation, Redmond, WA), and $P<0.05$ was considered significant. Confidence in- tervals for proportions were calculated according to the efficient-score method based on the Wilson estimate of population proportion, due to the situation of small numbers (Newcombe, 1998). Results were evaluated using Pearson's chi-squared test; however, if any value of a cell of the contingency table was $<5$, Fisher's exact test was applied instead.

A simulation analysis was conducted, which assumed that either 1,2 , or 5 shedding cows were present in the herd. Pools of 5 or 10 cows randomly selected from herds of 50 or 100 cows were simulated, with positive animals being randomly distributed across the pools (i.e., there was no systematic allocation of positive animals to specific pools). The probability of a pool being positive was computed based on proportion of individual positive samples within the pool (prp_pos) and whether the individual samples were slow (i.e., $>50$ d) to detect (slow) according to the following formula:

$$
\begin{aligned}
\ln \_ \text {odds } & =0.4916-(2.8746 \times \text { slow }) \\
+ & (3.4978 \times \text { prp_pos }) .
\end{aligned}
$$

The values for the 2 parameters in the model were determined from a logistic regression based on the 272 pools (of any size) that contained 1 or more positive individuals. The outcome of interest was whether or not the pool was positive on culture. For each pool, the proportion of positive animals in the pool (prp_pos) and whether or not all the positives in the pool were cultured at $>50 \mathrm{~d}$ (slow) were computed. The resulting model had a sensitivity of $87 \%$ and a specificity of $73 \%$. The estimates of the 2 effects included in the model were regarded as fixed effects in simulations. The simulation was set to contain 1,000 iterations and was tested for herds of 50 and 100, assuming all cows in the herd would be tested. The simulation also determined the probability that the shedding cows would be spread across multiple pools, or clustered among pools of 5 or 10 cows. The probabilities of possible patterns were determined, as well as the probability of a herd with that pooling pattern testing positive. A weighted mean herd probability was calculated for 1,2 , and 5 shedding cows to determine the average probability that the herd would have at least 1 test-positive pool.

\section{RESULTS}

\section{Culture of Individual Fecal Samples}

Of the 994 cows tested, 160 (16.1\%) were tissue culture-positive, whereas $36(3.6 \%)$ were fecal culturepositive. Of the 36 fecal culture-positive cows, 5 (3.1\%) were tissue culture-negative. 


\section{Culture of Pooled Fecal Samples}

In total, 840 pools were created of which 775 contained 1 or more tissue-culture positive cows (Table 1). Furthermore, 272-pooled samples contained feces from 1 or more fecal culture-positive cows, with 2 pools containing fecal culture-positive samples while not containing any tissue culture-positive cows.

Overall, a pool size of 5 had the highest sensitivity $(67 \%)$; however, this was not significantly different from the sensitivity assessed for pool sizes of 10 (59\%; Table 2). Pool sizes of 3, 8, and 15 had sensitivities of 47,44 , and $39 \%$, respectively, all significantly lower than the sensitivity for pool size of 5. Similarly, comparing sensitivities to identify 1 fecal culture-positive cow within each pool, pools of $5(62 \%)$ and $10(52 \%)$ had the highest sensitivity.

Sensitivity in pools of 3 increased significantly from 35 to $100 \%$ as the number of fecal culture-positive cows within the pools increased from 1 to 2 (Table 2). Although not statistically significant, the same trend was apparent in pool sizes of 5,8 and 10 , resulting in an overall significant difference in the sensitivity for pools containing 1 versus 2 individual culture-positive fecal samples (sensitivity of 44 versus $71 \%$, respectively). However, this trend did not continue when the number of positive samples within pools further increased to 3 and 4 . Overall sensitivity across pools with 3 and 4 fecal-culture positive cows was 63 and $60 \%$, respectively.

Pools containing early-detected individual samples had a higher probability of resulting in a positive pool in all pool sizes. The sensitivity to detect these early-detected individuals was highest in pools of 5 (85\%); however, this was not different from other pool sizes that contained 1 fast-detected positive individual sample (Table 3). Sample size numbers among the slow detection positive samples was low. Sensitivity to identify a positive pool that contained an individual sample that was detected after $50 \mathrm{~d}$ using the TREK ESP Culture System II was lower compared with pools that contained an early detected sample. This was the case across all pool sizes; however, pools of 5 containing 1 late-detected sample had the highest sensitivity (25\%), which was not statistically different from pools of 8 $(20 \%)$ or pools of $10(8 \%)$. None of the pools containing late-detected individual samples in pools of 3 and pools of 15 were detected as being positive.

\section{Herd Level Estimations}

The sensitivity for having at least 1 positive pool as determined by the herd test simulation using the sensitivity estimates for individual pools, was similar whether it was a 50- or 100-cow herd, assuming all

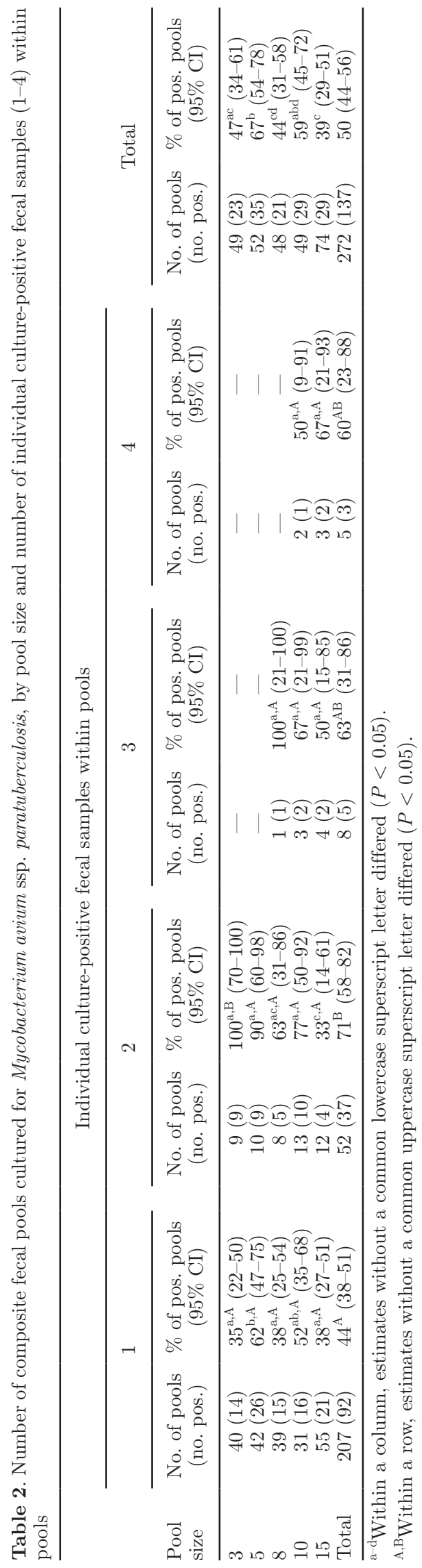

Journal of Dairy Science Vol. 101 No. 8, 2018 
Table 3. Distribution of pooled fecal samples (and sensitivity) for Mycobacterium avium ssp. paratuberculosis for pool with 1 positive sample, by pool size and time to positive detection of individual samples

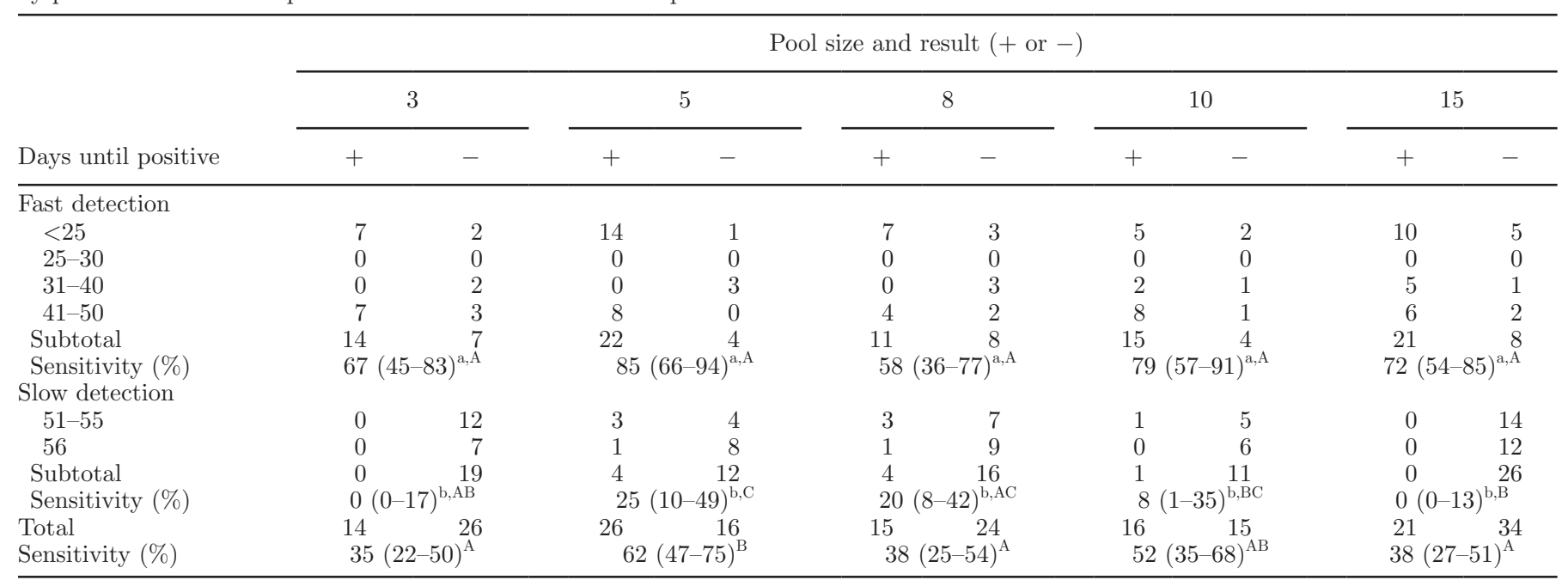

${ }^{\mathrm{a}, \mathrm{b}}$ Within a column, estimates without a common lowercase superscript letter differed $(P<0.05)$.

${ }^{\mathrm{A}-\mathrm{C}}$ Within a row, estimates without a common uppercase superscript letter differed $(P<0.05)$.

cows would be tested. As a result, only the 100-cow simulation is described (Table 4). Using pools of 5 that included 1, 2, or 5 fecal culture-positive cows, weighted herd sensitivity was 62,86 , and $99 \%$, respectively. Using pools of 10 with 1,2 , or 5 fecal culture-positive cows, the estimated sensitivity for having at least 1 positive pool was 52,76 , and $97 \%$, respectively (Table 4 ). For example, if fecal pooling using pools of 5 were to be performed to screen a herd of 100 cows that had 1 true fecal culture-positive cow, the probability of detecting the herd as positive would be $62 \%$. Testing the same herd with fecal pools of 10 , the probability of detecting the herd as positive would be $52 \%$. However, if 2 cows were positive, they could either be in the same pool or in 2 pools. The probability of determining the herd as positive in pools of 5 would be $86 \%$ if they were in 2 separate pools or $90 \%$ if they were in the same pool (the probability of both samples being in the same pool was $4 \%$; Table 4). The probability of determining the herd as positive in pools of 10 would be $76 \%$ if they were in 2 separate pools or $77 \%$ if they were in the same pool.

\section{DISCUSSION}

Sensitivities using the TREK ESP Culture System II were comparable with previous studies using this diagnostic approach or other laboratory tests (e.g., Tavornpanich et al., 2004; van Schaik et al., 2007; Wells et al., 2002a). Also, pooled fecal culture was a reasonably sensitive method of detecting fecal shedding cattle in various pool sizes. In this manner, sensitivity was defined as the ability of a MAP fecal-culture-positive cow within a pool to produce a positive pooled sample.
The difference in sensitivity across pool sizes was surprisingly small, ranging from $39 \%$ in pools of 15 to $67 \%$ in pools of 5 . Although this difference was statistically significant, overall, pool size did not affect the sensitivity as much as expected.

In addition to the influence of pool size, detection sensitivity of pooled fecal culture has also been associated with concentration of bacteria in the infected sample (Wells et al., 2002a). Results from pooling of feces from 10 cows indicated that, compared with concurrent bacterial culture of individual infected samples, 37 to $44 \%$ of pooled samples with low bacterial concentrations yielded positive culture results and 94\% of pooled samples with high bacterial concentrations yielded positive results (Wells et al., 2002a). In the current study, the surprisingly low sensitivity of pools of 3 cows with 1 fecal culture-positive sample included could be explained if the positive samples were low fecal shedders and less likely to yield a positive pool.

Using time until detection as a proxy for heavy shedders has not been extensively studied; however, it has been established that the correlation between these 2 variables is high (Stich et al., 2004). The low sensitivity in the pools of 3 was likely influenced by having a certain positive cow present in 5 of the negative pools of 3 . This cow was also the sole positive sample in 5 other pools $(2$ in pools of 8,1 in 10, and 2 in 15) and never triggered a positive pool. Across all pools, sensitivity dramatically increased when only pools containing early detectors (i.e., within the first $50 \mathrm{~d}$ ) were considered.

Application of diagnostic tests is an integral part of attempts to control JD at the herd level (Barkema et al., 2017). Using ELISA for herd-level diagnosis is prob- 
lematic in low-prevalence herds (Wells et al., 2002b); however, progress can be made using ELISA in higherprevalence herds (Jubb and Galvin, 2000; Barkema et al., 2017). That, in part, was the rationale in using pooled testing strategies when MAP prevalence is low. Maximizing economic benefit without sacrificing diagnostic sensitivity is the ultimate goal of pooled sample testing. This study provided a unique opportunity to examine various pool sizes on samples that were randomly allocated and not strategically constructed based on either age or colony counts, providing a legitimate model to resemble herd testing. A simulation study to estimate costs and benefits of whole-herd testing in low-prevalence herds by use of pooled fecal samples in cattle revealed that pooling reduced costs by $43 \%$ in a 100-cow herd and $71 \%$ in a 1,000-cow herd (van Schaik et al., 2003). Similar results were documented for pooled fecal culture in sheep flocks, where it was reported that the laboratory cost of pooled samples applied as a flock test was approximately 30\% that of serologic testing (Whittington et al., 2000).

The simulation model treated estimates of effects of the proportion of positive cows in the pool and the possibility that the pool contained only slow to detect animals were treated as fixed effects (i.e., no uncertainty in the estimates was incorporated into the analyses). Therefore, results are most applicable to the study population and more variability would be expected if those effects were allowed to vary across simulations. The simulation study demonstrated that probability of detecting positive pools did not depend on herd size (comparing herd sizes of 50 and 100 cows). Similarly, Messam et al. (2010) concluded that optimal pool size was independent of herd size when applying a Monte Carlo simulation model to herd sizes of $300,1,000$, and 3,000 cows. In our simulation model, sensitivities were relatively high, especially for apparent prevalences of 2 and $5 \%$. Weighted mean sensitivities were slightly higher in pools of 5 ; however, the probability of detecting low-prevalence herds as positive was relatively high (52-99\%) whether pools of 5 or 10 were used. Similarly, culture of fecal samples in composite pools of 5 and 10 cows resulted in herd level sensitivities that were not different, consistent with findings from van Schaik et al. (2007), who used a modified Herrold's egg yolk medium to culture fecal pools. There was an economic benefit of using pools of 10 over 5 in a herd of 300 cows and a prevalence of $5 \%$, even when costs for follow-up and identification of individual cows were considered (van Schaik et al., 2007). Based on estimates of sensitivity for pools of 3 in this study, there appeared to be no substantial diagnostic benefit of using pools of 3 and therefore it would be more economical to use larger pools.

\section{CONCLUSIONS}

Overall, culturing pools of 5 or 10 had similar diagnostic sensitivity within the broth culture system. In conjunction with economic studies, we inferred that

Table 4. Simulation of possible outcomes of testing for Mycobacterium avium ssp. paratuberculosis in a 100-cow herd using fecal pools of 5 and 10 , assuming 1,2 , or 5 cows in the herd were truly positive

\begin{tabular}{|c|c|c|c|c|c|}
\hline Pool size & $\begin{array}{c}\text { No. of } \\
\text { positive cows }\end{array}$ & & $\begin{array}{l}\text { Probability of } \\
\text { occurrence (\%) }\end{array}$ & $\begin{array}{l}\text { Probability of a } \\
\text { positive test }^{1}(\%)\end{array}$ & $\begin{array}{l}\text { Weighted mean } \\
\text { herd probability }(\%)\end{array}$ \\
\hline \multirow[t]{6}{*}{5} & 1 & 1 per pool & 100 & 62 & 62 \\
\hline & 2 & 1 per pool & 96 & 86 & 86.1 \\
\hline & 5 & 1 per pool & 64.3 & 99.2 & 99.3 \\
\hline & & $2+1+1+1$ & 32.5 & 99.4 & \\
\hline & & $2+2+1$ & 2.4 & 99.6 & \\
\hline & & 5 & $-^{3}$ & $100^{2}$ & \\
\hline \multirow[t]{5}{*}{10} & 1 & 1 per pool & 100 & 52 & 52 \\
\hline & 2 & 1 per pool & 83 & 76 & 76.1 \\
\hline & & 2 in one pool & 17 & 77 & \\
\hline & 5 & 1 per pool & 33.4 & 97.2 & 97.0 \\
\hline & & $2+1+1+1$ & 51.4 & 97.4 & \\
\hline
\end{tabular}

${ }^{1}$ Probabilities determined from sensitivities in Table 2 .

${ }^{2}$ Assuming the probability was equal to the next lowest available estimate.

${ }^{3}$ Missing values occurred if probability was very close to zero. 
it would be justifiable to use pools of 10 . Considering no diagnostic test is infallible at the herd level, pooled fecal culture performed similar to other diagnostic modalities. Potential limitations of pooled samples include thorough mixing and low shedders. However, the economic benefits of pooled culture along with its similar diagnostic abilities made it a reasonable economic choice for herd-level testing.

\section{ACKNOWLEDGMENTS}

This study was made possible with support from Dairy Farmers of Canada (Ottawa, ON, Canada) and the Natural Sciences Engineering and Research Council (NSERC; Ottawa, ON, Canada). Ritter and Barkema are supported by NSERC's Industrial Research Chair Program with industry contributions from Alberta Milk (Edmonton, AB, Canada), Dairy Farmers of Canada (Ottawa, ON, Canada), Dairy Farmers of Manitoba (Winnipeg, MB, Canada), British Columbia Dairy Association (Burnaby, BC Canada), Westgen Endowment Fund (Milner, BC, Canada), Canadian Dairy Network (Guelph, ON, Canada), and CanWest DHI (Guelph, ON, Canada). We also thank John Kastelic (University of Calgary) for editing the manuscript and Theresa Andrews, Lloyd Dalziel, and Ricky Milton (University of Prince Edward Island) for assistance with fecal sample collection.

\section{REFERENCES}

Barkema, H. W., S. Hendrick, J. de Buck, G. G. Kaplan, and K. Rioux. 2010. Crohn's disease in humans and Johne's disease in cattle-Linked diseases? Pages 197-213 in Zoonotic Pathogens in the Food Chain. D. Krause and S. Hendrick, ed. CAB International, Wallingford, UK.

Barkema, H. W., K. Orsel, S. S. Nielsen, A. P. Koets, V. P. Rutten, J. P. Bannantine, G. P. Keefe, D. F. Kelton, S. J. Wells, R. J. Whittington, C. G. Mackintosh, E. J. Manning, M. F. Weber, C. Heuer, T. Forde, C. Ritter, S. Roche, C. Corbett, R. Wolf, P. Griebel, J. P. Kastelic, and J. De Buck. 2017. Knowledge gaps that hamper prevention and control of Mycobacterium avium subspecies paratuberculosis infection. Transbound. Emerg. Dis. https://doi.org/10 $.1111 /$ tbed.12723.

Garcia, A. B., and L. Shalloo. 2015. Invited review: The economic impact and control of paratuberculosis in cattle. J. Dairy Sci. 98:5019-5039.

Jubb, T., and J. Galvin. 2000. Herd testing to control bovine Johne's disease. Vet. Microbiol. 77:423-428.

Kalis, C. H. J., J. W. Hesselink, H. W. Barkema, and M. T. Collins. 2000. Culture of strategically pooled bovine fecal samples as a method to screen herds for paratuberculosis. J. Vet. Diagn. Invest. 12:547-551.

Kim, S. G., E. H. Kim, C. J. Lafferty, L. J. Miller, H. J. Koo, S. M. Stehman, and S. J. Shin. 2004. Use of conventional and realtime polymerase chain reaction for confirmation of Mycobacterium avium ssp. paratuberculosis in a broth-based culture system ESP II. J. Vet. Diagn. Invest. 16:448-453.

McKenna, S. L., G. P. Keefe, H. W. Barkema, J. McClure, J. A. Vanleeuwen, P. Hanna, and D. C. Sockett. 2004. Cow-level prevalence of paratuberculosis in culled dairy cows in Atlantic Canada and Maine. J. Dairy Sci. 87:3770-3777.

McKenna, S. L., G. P. Keefe, A. Tiwari, J. VanLeeuwen, and H. W. Barkema. 2006. Johne's disease in Canada part II: disease impacts, risk factors, and control programs for dairy producers. Can. Vet. J. 47:1089-1099.

Messam, L. L., J. M. O'Brien, S. K. Hietala, and I. A. Gardner. 2010. Effect of changes in testing parameters on the cost-effectiveness of two pooled test methods to classify infection status of animals in a herd. Prev. Vet. Med. 94:202-212.

Murray, P. R. 1999. Manual of Clinical Microbiology. 7th ed. Am. Society Micro Press, Washington, DC.

Newcombe, R. G. 1998. Two-sided confidence intervals for the single proportion: Comparison of seven methods. Stat. Med. 17:857-872.

Poupart, P., M. Coene, H. Van Heuverswyn, and C. Cocito. 1993 Preparation of a specific RNA probe for detection of Mycobacterium paratuberculosis and diagnosis of Johne's disease. J. Clin. Microbiol. 31:1601-1605.

Stich, R. W., B. Byrum, B. Love, N. Theus, L. Barber, and W. P. Shulaw. 2004. Evaluation of an automated system for non-radiometric detection of Mycobacterium avium paratuberculosis in bovine feces. J. Microbiol. Methods 56:267-275.

Tavornpanich, S., I. A. Gardner, R. J. Anderson, S. Shin, R. H. Whitlock, T. Fyock, J. M. Adaska, R. L. Walker, and S. K. Hietala. 2004. Evaluation of microbial culture of pooled fecal samples for detection of Mycobacterium avium subsp paratuberculosis in large dairy herds. Am. J. Vet. Res. 65:1061-1070.

van Schaik, G., F. M. Pradenas, N. A. Mella, and V. J. Kruze. 2007. Diagnostic validity and costs of pooled fecal samples and individual blood or fecal samples to determine the cow- and herd-status for Mycobacterium avium ssp. paratuberculosis. Prev. Vet. Med. 82:159-165.

van Schaik, G., S. M. Stehman, Y. H. Schukken, C. R. Rossiter, and S. J. Shin. 2003. Pooled fecal culture sampling for Mycobacterium avium ssp. paratuberculosis at different herd sizes and prevalence. J. Vet. Diagn. Invest. 15:233-241.

Vary, P. H., P. R. Andersen, E. Green, J. Hermon-Taylor, and J. J. McFadden. 1990. Use of highly specific DNA probes and the polymerase chain reaction to detect Mycobacterium paratuberculosis in Johne's disease. J. Clin. Microbiol. 28:933-937.

Vialard, J., A. Lacheretz, A. Thiercy, Y. Richard, and M. Prave. 1993 Detection of bovine paratuberculosis by culture of pooled feces. Rev. Med. Vet. 144:527-533.

Weber, M. F., H. Groenendaal, H. J. W. van Roermund, and M. Nielen. 2004. Simulation of alternatives for the Dutch Johne's disease certification-and-monitoring program. Prev. Vet. Med. 62:1-17.

Wells, S. J., S. M. Godden, C. J. Lindeman, and J. E. Collins. 2003. Evaluation of bacteriologic culture of individual and pooled fecal samples for detection of Mycobacterium paratuberculosis in dairy cattle herds. J. Am. Vet. Med. Assoc. 223:1022-1025.

Wells, S. J., R. H. Whitlock, C. J. Lindeman, and T. Fyock. 2002a. Evaluation of bacteriologic culture of pooled fecal samples for detection of Mycobacterium paratuberculosis. Am. J. Vet. Res. 63:1207-1211.

Wells, S. J., R. H. Whitlock, B. A. Wagner, J. Collins, F. Garry, H. Hirst, J. Lawrence, W. J. Saville, and A. L. Naugle. 2002b. Sensitivity of test strategies used in the Voluntary Johne's Disease Herd Status Program for detection of Mycobacterium paratuberculosis infection in dairy cattle herds. J. Am. Vet. Med. Assoc. 220:1053-1057.

Whitlock, R. H., S. J. Wells, R. W. Sweeney, and J. Van Tiem. 2000. ELISA and fecal culture for paratuberculosis (Johne's disease): Sensitivity and specificity of each method. Vet. Microbiol. 77:387398.

Whittington, R. J., S. Fell, D. Walker, S. McAllister, I. Marsh, E Sergeant, C. A. Taragel, D. J. Marshall, and I. J. Links. 2000. Use of pooled fecal culture for sensitive and economic detection of $M y$ cobacterium avium ssp. paratuberculosis infection in flocks of sheep. J. Clin. Microbiol. 38:2550-2556. 